24. S. Hammes-Schiffer, S. ]. Benkovic, Annu. Rev. Biochem. 75, 519 (2006).

25. E. Z. Eisenmesser et al., Nature 438, 117 (2005).

26. S. Kumar, B. Y. Ma, C. ]. Tsai, N. Sinha, R. Nussinov, Protein Sci. 9, 10 (2000).

27. R. Koradi, M. Billeter, K. Wüthrich, J. Mol. Graph. 14, 51 (1996).

28. N. R. Skrynnikov, F. W. Dahlquist, L. E. Kay, J. Am. Chem. Soc. 124, 12352 (2002).
29. We thank ]. Chung and G. Kroon for assistance with the NMR experiments, L. Tennant for technical assistance, and S. Benkovic for valuable discussions. This work was supported by NIH grants GM56879 and GM75995 and by the Skaggs Institute for Chemical Biology. D.D.B. was a recipient of a Canadian Institutes of Health Research postdoctoral fellowship. D.M. was supported by a postdoctoral fellowship from NIH.
Supporting Online Material

www.sciencemag.org/cgi/content/full/313/5793/1638/DC1

Materials and Methods

Figs. S1 to S11

Tables S1 to S4

22 May 2006; accepted 25 July 2006

10.1126/science. 1130258

\section{Imaging Intracellular Fluorescent Proteins at Nanometer Resolution}

Eric Betzig, ${ }^{1,2 *} \dagger$ George H. Patterson, ${ }^{3}$ Rachid Sougrat, ${ }^{3}$ O. Wolf Lindwasser, ${ }^{3}$ Scott Olenych, ${ }^{4}$ Juan S. Bonifacino, ${ }^{3}$ Michael W. Davidson, ${ }^{4}$ Jennifer Lippincott-Schwartz, ${ }^{3}$ Harald F. Hess ${ }^{5 *}$

We introduce a method for optically imaging intracellular proteins at nanometer spatial resolution. Numerous sparse subsets of photoactivatable fluorescent protein molecules were activated, localized (to $\sim 2$ to 25 nanometers), and then bleached. The aggregate position information from all subsets was then assembled into a superresolution image. We used this method-termed photoactivated localization microscopy-to image specific target proteins in thin sections of lysosomes and mitochondria; in fixed whole cells, we imaged vinculin at focal adhesions, actin within a lamellipodium, and the distribution of the retroviral protein Gag at the plasma membrane.

$\mathrm{T}$ ransfected cells expressing fluorescent proteins (1) contain information that is accurate at the molecular level about the spatial organization of the target proteins to which they are bound. However, the best resolution that can be obtained by diffractionlimited conventional optical techniques is coarser than the molecular level by two orders of magnitude. Great progress has been made with superresolution methods that penetrate beyond this limit, such as near field (2), stimulated emission depletion (3), structured illumination $(4,5)$, and reversible saturable optical fluorescence transitions microscopy ( 6 ), but the goal remains a fluorescence technique capable of achieving resolution closer to the molecular scale.

Early results (7) in single-molecule microscopy (8) and the spatiospectral isolation of individual exciton recombination sites in a semiconductor quantum well (9) led to a proposal for a means of molecular resolution fluorescence microscopy a decade ago (10). In brief, individual molecules densely packed within the resolution limit of a given instrument [as defined by its point-spread function (PSF)] are first isolated from one another on the basis of one or more distinguishing optical characteristics. Each molecule is then localized to much higher precision by determining its center of fluorescence emission through a statistical fit of

\footnotetext{
${ }^{1}$ Howard Hughes Medical Institute, Janelia Farm Research Campus, Ashburn, VA 20147, USA. ${ }^{2}$ New Millennium Research, LLC, Okemos, MI 48864, USA. ${ }^{3}$ Cell Biology and Metabolism Branch, National Institute of Child Health and Human Development (NICHD), Bethesda, MD 20892, USA ${ }^{4}$ National High Magnetic Field Laboratory, Florida State University, Tallahassee, FL 32310, USA. ${ }^{5}$ NuQuest Research, LLC, La Jolla, CA 92037, USA.

*These authors contributed equally to this work. †To whom correspondence should be addressed. E-mail: betzige@janelia.hhmi.org.
}

the ideal PSF to its measured photon distribution (Fig. 1). When the background noise is negligible compared with the molecular signal, the error in the fitted position is $\sigma_{x, y} \approx s /\left(N^{1 / 2}\right)$, where $s$ is the standard deviation of a Gaussian approximating the true PSF $\approx 200 \mathrm{~nm}$ for light of wavelength $\lambda=500 \mathrm{~nm}$ ) and $N$ is the total number of detected photons $(11,12)$. Given that it is possible to detect many more than $10^{4}$ photons from a single fluorophore before it bleaches, single-molecule localization to nearly 1-nm precision has already been demonstrated (13-15) and applied to studies of molecular motor dynamics (13).

Multiple emitters within a single diffractionlimited region (DLR) have been isolated from one another by either spectral $(15,16)$ or temporal means, the latter exploiting the photobleaching $(14,17)$ or blinking $(18)$ of the emitters. However, the number of emitters isolated per DLR (typically 2 to 5 ) has been too small to give resolution within the DLR that is comparable to existing superresolution techniques, and it is far from the molecular level. Here, we developed a method for isolation of single molecules at high densities (up to $\sim 10^{5} / \mu \mathrm{m}^{2}$ ) based on the serial photoactivation and subsequent bleaching of numerous sparse subsets of photoactivatable fluorescent protein (PA-FP) molecules (19-24) within a sample. We then applied the method to image specific target proteins in thin $(\sim 50$ - to $80-\mathrm{nm})$ sections and near the surfaces of fixed cultured cells, resolving the most precisely localized molecules therein at separations $(\sim 10 \mathrm{~nm})$ approaching the molecular level.

The method and typical data subsets are shown in Fig. 1. Cultured mammalian cells expressing PA-FP-tagged target proteins were prepared by transient transfection, fixed, and processed on cover slips either as whole cells or in cryosections cut from a centrifuged pellet of cells (25). Such cover slips were then placed in a custom microscope chamber (fig. S1) designed to minimize thermal and mechanical drift (fig. S2) (25). They were continuously excited by a laser at a wavelength $\left(\lambda_{\text {exc }}=561\right.$ $\mathrm{nm}$ ) near the excitation maximum of the activated form of the expressed PA-FPs. Finally, to minimize both autofluorescence and detector noise, they were imaged by total internal reflection fluorescence (TIRF) microscopy $(13,26)$ onto an electron-multiplying charge coupled device (EMCCD) camera that can detect single photons.

Initial image frames typically consisted of sparse fields of individually resolvable single molecules on a weaker background presumably dominated by the much larger population of PA-FP molecules still in the inactivated state. When necessary, excitation and thus bleaching was maintained until such sparse fields were obtained. Additional image frames were then captured until single-molecule bleaching resulted in a mean molecular separation considerably larger than that required for isolation (Fig. 1, A and C). At that point, we applied a light pulse from a second laser at a wavelength $\left(\lambda_{\text {act }}=405 \mathrm{~nm}\right)$ capable of activating the remaining inactive PA-FPs, at a duration and intensity chosen so that the overall density of activated PA-FPs was increased back to a higher, but still resolvable, level (Fig. 1, B and D). This process of photoactivation, measurement, and bleaching was then repeated (movie S1) for many cycles over $\sim 10^{4}$ to $>10^{5}$ image frames (depending on the expression level and spatial distribution of the PA-FPs) until the population of inactivated, unbleached molecules was depleted. At typical frame rates of $\sim 0.5$ to $1.0 \mathrm{~s}$, between 2 and 12 hours were required to acquire a complete image stack that could be distilled to a single superresolution image containing $\sim 10^{5}$ to $>10^{6}$ localized molecules. We continued to explore methods (such as brighter molecules, higher excitation power, and higher activation density) to speed this process.

When the $x y$ frames from any such image stack are summed across time $t$, the molecular signals overlap to produce a diffraction-limited image (Fig. 1, E and F) similar to that obtained by conventional TIRF, in which all molecules emit simultaneously (fig. S3). However, when the data are plotted in a multidimensional volume xyt (Fig. 1, center), the signal from each molecule $m$ is uniquely isolated and can be summed at each pixel and across all of 
the frames in which it appears. This result (Fig. $1 \mathrm{G}$, left) is then fitted using a robust nonlinear least squares algorithm to an assumed Gaussian PSF of free center coordinates $x_{\mathrm{o}}, y_{\mathrm{o}}$ (Fig. 1G, center) (25), yielding coordinates $x_{\mathrm{m}}, y_{\mathrm{m}}$ for the location of the molecule, with a position uncertainty $\left(\sigma_{x, y}\right)_{\mathrm{m}}$. Finally, each molecule is rendered in a new $x y$ frame as a Gaussian of standard deviation $\left(\sigma_{x, y}\right)_{\mathrm{m}}$ (rather than the much larger standard deviation $s$ of the original PSF), centered at $x_{\mathrm{m}}, y_{\mathrm{m}}$ (Fig. 1, G, right, and $\mathrm{A}^{\prime}$ to $\mathrm{D}^{\prime}$ ) and normalized to unit strength when integrated over all $x y$ space. Thus, the super-

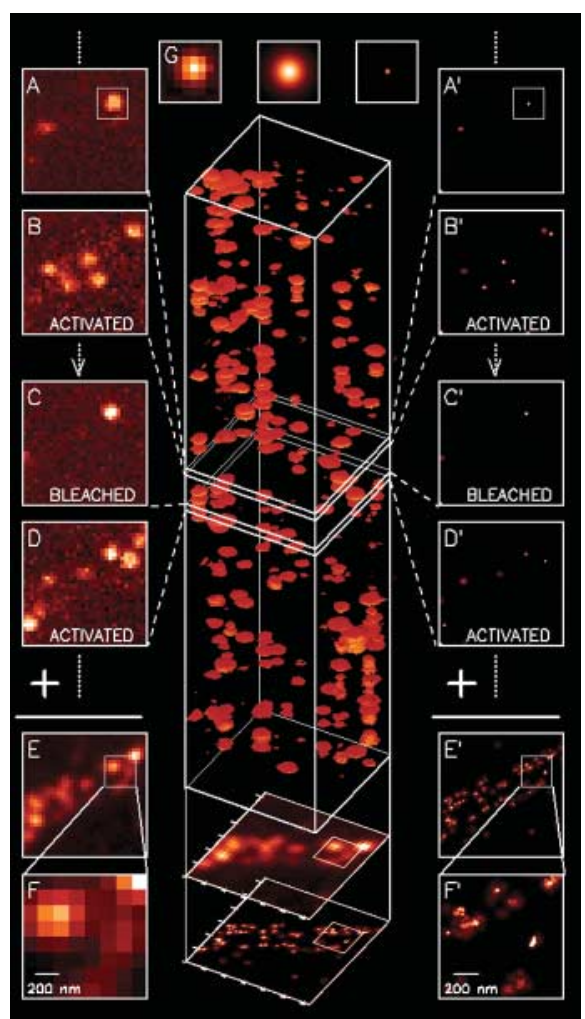

Fig. 1. The principle behind PALM. A sparse subset of PA-FP molecules that are attached to proteins of interest and then fixed within a cell are activated ( $\mathbf{A}$ and B) with a brief laser pulse at $\lambda_{\text {act }}=405 \mathrm{~mm}$ and then imaged at $\lambda_{\text {exc }}=561 \mathrm{~mm}$ until most are bleached (C). This process is repeated many times (C and D) until the population of inactivated, unbleached molecules is depleted. Summing the molecular images across all frames results in a diffraction-limited image (E and $\mathbf{F}$ ). However, if the location of each molecule is first determined by fitting the expected molecular image given by the PSF of the microscope $[(\mathbf{G})$, center] to the actual molecular image [(G), left], the molecule can be plotted [(G), right] as a Gaussian that has a standard deviation equal to the uncertainty $\sigma_{x, y}$ in the fitted position. Repeating with all molecules across all frames ( $\mathbf{A}^{\prime}$ through $\mathbf{D}^{\prime}$ ) and summing the results yields a superresolution image ( $\mathbf{E}^{\prime}$ and $\mathbf{F}^{\prime}$ ) in which resolution is dictated by the uncertainties $\sigma_{x, y}$ as well as by the density of localized molecules. Scale: $1 \times 1 \mu \mathrm{m}$ in (F) and $\left(\mathrm{F}^{\prime}\right), 4 \times 4 \mu \mathrm{m}$ elsewhere. resolution image obtained by summing the rendered Gaussians associated with all localized molecules in the original image stack (Fig. 1, E' and $F^{\prime}$ ) provides a probability density map where brightness is proportional to the likelihood that a PA-FP molecule can be found at a given location.

This technique, termed photoactivated localization microscopy (PALM), is capable of resolving the most precisely localized molecules at separations of a few nanometers. These represent the very brightest emitters (the much larger population of all isolated molecules exhibits a much broader range of photon counts; fig. S4). Thus, when rendering PALM images, a fundamental trade-off exists: Including fewer, but brighter, molecules results in higher localization and crisper images, but at a reduced molecular density giving less complete information about the spatial distribution of the target protein (fig. S5). Both parameterslocalization precision and the density of rendered molecules - are key to defining performance in PALM. Their specific values for the images in Figs. 2 to 4 are given in table S1.

This performance is largely dictated by the photophysical characteristics of the PA-FPs.
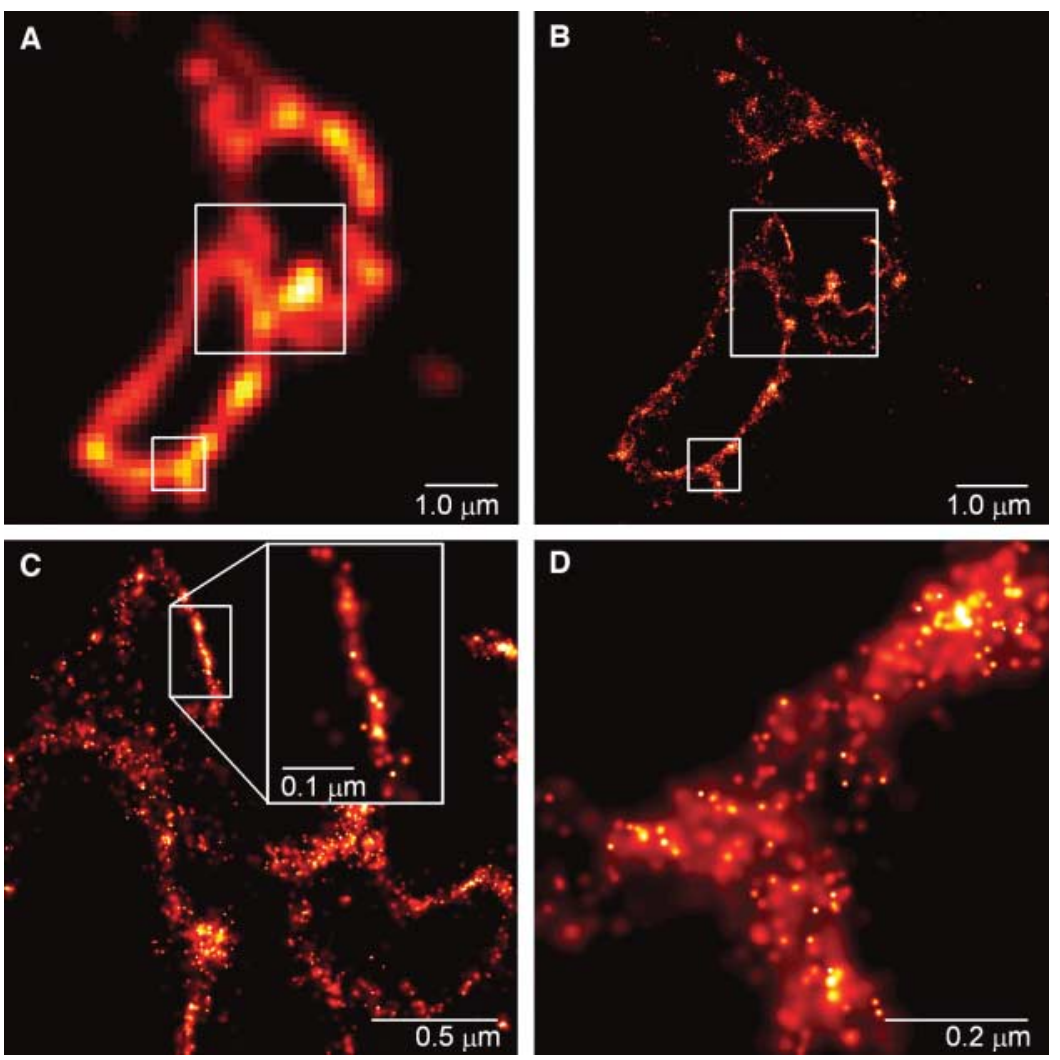

Fig. 2. Comparative summed-molecule TIRF (A) and PALM (B) images of the same region within a cryoprepared thin section from a COS-7 cell expressing the lysosomal transmembrane protein CD63 tagged with the PA-FP Kaede. The larger boxed region in (B), when viewed at higher magnification (C) reveals smaller associated membranes that may represent interacting lysosomes or late endosomes that are not resolvable by TIRF. In a region where the section is nearly orthogonal to the lysosomal membrane, the most highly localized molecules fall on a line of width $\sim 10 \mathrm{~nm}$ (inset). In an obliquely cut region [(D), from the smaller boxed region in (B)], the distribution of CD63 within the membrane plane can be discerned.
Longer photobleaching half-life leads to more photons per molecule, but for a given excitation intensity, it also requires longer data acquisition times between activation pulses to maintain an appropriate density of individually resolv$\sigma$ and fluores. Higher excitation cross-sectional speed this process of signal extraction and bleaching, with the added benefit of increasing the molecular contrast relative to the autofluorescence background. Also vital is the contrast $C\left(\lambda_{\text {exc }}\right)=(\sigma \Phi)_{\text {act }} /(\sigma \Phi)_{\text {inact }}$ between the PA-FP ins activated and inactivated state at $\lambda_{\text {exc }}$ density beyond which the background from many weakly emitting inactivated molecules in a DLR dominates the signal from a single activated one. PA-FPs that remain activated until ensure that all possible photons are . Finally, PA-FPs less prone to blinking distinguishe, given that it can be difficult to multiple molecules that are serially activated and bleached in the same DLR (25).

Although we have demonstrated isolation and localization with both green [photoactivatable green fluorescent protein (PA-GFP) and Dronpa] 
and yellow [Kaede, Kikume Green-Red (KikGR), and Eos Fluorescent Protein (EosFP)] excitable PA-FPs, for imaging cellular structures we focused on tetrameric Kaede and the various oligomers of EosFP - the former for its somewhat higher brightness and the latter for their less perturbative effect on cellular structure and function. Each also exhibits high contrast relative to both the inactivated state and autofluorescence background at $\lambda_{\text {exc }}=561 \mathrm{~nm}$.

We used PALM imaging to view intracellular structures in thin cryosections (25), akin to those used in transmission electron microscopy (TEM) but imaged under ambient conditions (Figs. 2 and 3). In Fig. 2, lysosomes in a COS-7 cell are visualized through expression of the lysosomal transmembrane protein CD63 fused to Kaede. Localization to the lysosome membrane was confirmed by comparative immunofluorescence labeling in similarly prepared samples (fig. S6). A TIRF image shows the outlines of the limiting membrane (Fig. 2A) but only hints at the intricate structure that is resolved by PALM, such as smaller associated membranes that may represent interacting lysosomes or late endosomes (Fig. 2, B and C). Indeed, in regions where the section plane is nearly orthogonal to the membrane, the most highly localized molecules fall on a line with an apparent width of $\sim 10 \mathrm{~nm}$ (inset, Fig. 2C), demonstrating that they are indeed fixed and that sample drift has been successfully mitigated (25). In other regions of the cryosection where the cut is more oblique to the lysosome, a wider, yet still sharply defined, swath of membrane is projected onto the image plane (Fig. 2D), permitting detailed investigation of the distribution of CD63 within the membrane plane.

In Fig. 3, PALM images of dEosFP-tagged cytochrome-C oxidase import sequence localized within the matrix of mitochondria in a COS-7 cell are compared with TEM images of the same mitochondria. The high degree of correlation between the two data sets validates the PALM imaging principle, and the sharpness of the mitochondrial edges (Fig. $3 \mathrm{H}$ ) as viewed by PALM is far closer to that seen by TEM than that observed by diffraction-limited TIRF (Fig. 3A). Such comparative PALM/TEM imaging permits the nanometer-scale distribution of a specified protein to be determined in relation to the rest of the cellular ultrastructure at much higher molecular density than in immunolabeled TEM - more than 5500 molecules are localized in Fig. 3E, compared with the 20 or so particles typical in immunogold labeling of the mitochondrial matrix. Superposition of the PALM and TEM images (Fig. 3, D and G) also reveals that the matrix reporter molecules extend up to, but not into, the $\sim 20$-nm outer mitochondrial membrane, underscoring the resolution capability of the technique. Correlated PALM/TEM does not have the added preparation steps and specificity issues associated with exogenous labels for combined
fluorescence/EM such as fluorescein or resorufin arsenical helix binder (27). Finally, efforts are underway to establish dual-labeled PALM or PALM fluorescence resonance energy transfer, which would permit the relative distribution or regions of interaction between multiple proteins to be discerned at the nanometer level.

Thin sections are advantageous for PALM because they exhibit less autofluorescence than bulk samples, ensure that the PA-FPs are immobile, and permit the study of intracellular organelles that are inaccessible under TIRF excitation. However, demonstration of PALM on fixed cultured cells in phosphate-buffered saline (Fig. 4) is also notable both as a means to study proteins at or near the plasma membrane under minimally invasive conditions and as a precursor to eventual three-dimensional (3D) PALM imaging.

Confirmation that the nanometer-level resolution of PALM is retained under such conditions is given by the comparison of TIRF (Fig. 4A) and PALM (Fig. 4B) images of dEos-fused vinculin at focal adhesion regions (fig. S7) of a fox lung fibroblast (FoLu) cell to a cover slip. PALM reveals the heterogeneity within a selected attachment (box, Fig. 4A) and, in one subregion, suggests the partial assembly of a vinculin network (arrows, Fig. 4B). Similarly, a TIRF image (Fig. 4C) of tandem-dimer EosFP-fused actin in a cultured FoLu cell (fig. S8) shows both large cytoskeletal stress fibers and a lamellipodium, whereas PALM within the latter (Fig. 4D) reveals an increased concentration of actin at the leading
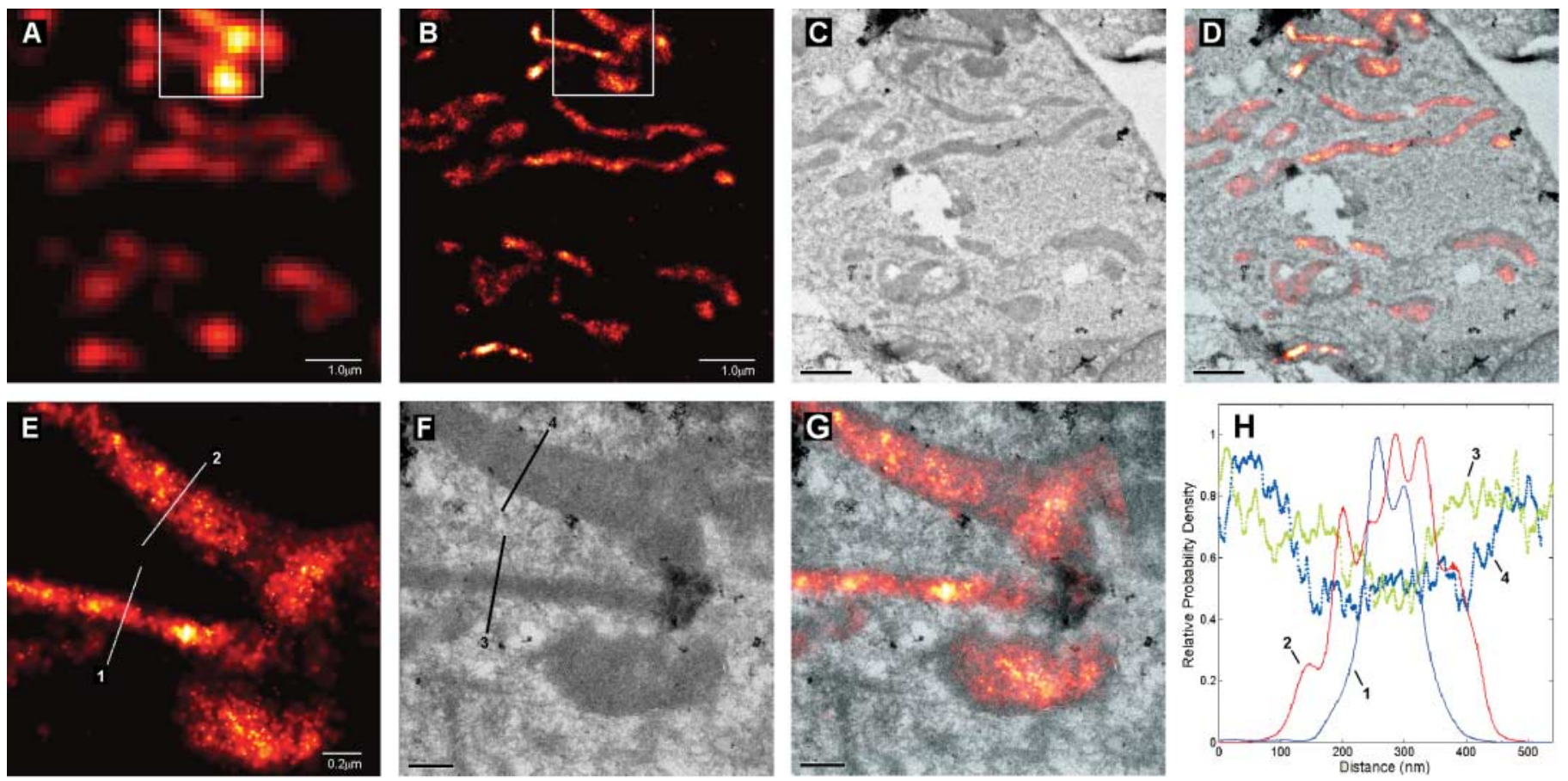

Fig. 3. Comparative summed-molecule TIRF (A), PALM (B), TEM (C), and PALM/TEM overlay (D) images of mitochondria in a cryo-prepared thin section from a COS-7 cell expressing dEosFP-tagged cytochrome-C oxidase import sequence. Higher magnification PALM (E), TEM (F), and overlay (G) images within the box in (B) reveal that these matrix re-

porter molecules extend up to, but not into, the $\sim 20$-nm outer mitochondrial membrane. The molecular distribution across two mitochondria along lines 1 and 2 in PALM image (E) are compared in (H) to the TEM signal along lines 3 and 4 in $(F)$ across the same mitochondria. Scale bars: $1.0 \mu \mathrm{m}$ in (A) to (D); $0.2 \mu \mathrm{m}$ in (E) to (G). 
edge. Under even higher magnification (inset, Fig. 4D), numerous short filaments are observed. These may be independent structures fixed in the process of assembly, or they may be part of a larger, continuous 3D network only partially revealed by the short extent of the evanescent excitation field.

In whole cells, PALM with TIRF excitation is well suited to studies of proteins bound to the plasma membrane, such as the dEosFP-fused Gag protein of human immunodeficiency virus 1 imaged by TIRF and PALM in Fig. 4, E and F, respectively. Gag, a retroviral protein that mediates the assembly of virus-like particles (VLPs), is revealed by PALM in various stages of organization: voids (arrows marked V), one high-density region (arrow R), and several tight clusters probably indicative of budding VLPs (arrows marked $\mathrm{P}$, and magnified inset of Fig. 4F).

In the future, PALM should benefit from improvements in and additions to the palette of available PA-FPs, as well as from the discovery of means to modify the PA-FP environment to enhance photostability (13) and suppress blinking. Recently, we demonstrated photoactivation in PALM through ultraviolet-induced uncaging (28) of fluorophores (fig. S10) which, when combined with immunolabeling or other developing methods to achieve high-specificity intracellular protein labeling $(27,29)$, might offer a different avenue to improved localization precision and faster frame rates, given that a broad spectrum of high-brightness caged fluorophores is potentially available.

Algorithmically, additional well-localized molecules might be mined from the data if better means are found to unambiguously collate the multiple photon bursts from blinking molecules. Possible improvements to the fitting algorithm to achieve higher localization accuracy should also be explored. Although most of the observed molecules are well represented by a circularly symmetric Gaussian PSF, possible systematic position errors due to chromophore orientation, pixel nonuniformity, and chromatic aberration deserve closer attention. Perhaps most importantly, position error due to background nonuniformity within the molecular fitting window needs to be addressed, particularly when the number of inactivated molecules contributing to this background is high.

Experimentally, multiple angles and polarizations of TIRF excitation may eventually permit the precise determination of the $x y z$ position and dipole orientation for fixed PA-FP molecules within the evanescent field. Standing wave TIRF could provide an excitation PSF of width $\sim \lambda_{\text {exc }} / 6$, improving localization precision for a given photon count. Bulk cellular autofluorescence complicates the extension of PALM to 3D, but the improved single-molecule sensitivity predicted for a proposed optical lattice microscope (30) may help. However, the most promising path to $3 \mathrm{D}$ may involve cryogenic PALM of vitrified cells, due to the narrow molecular line widths, large
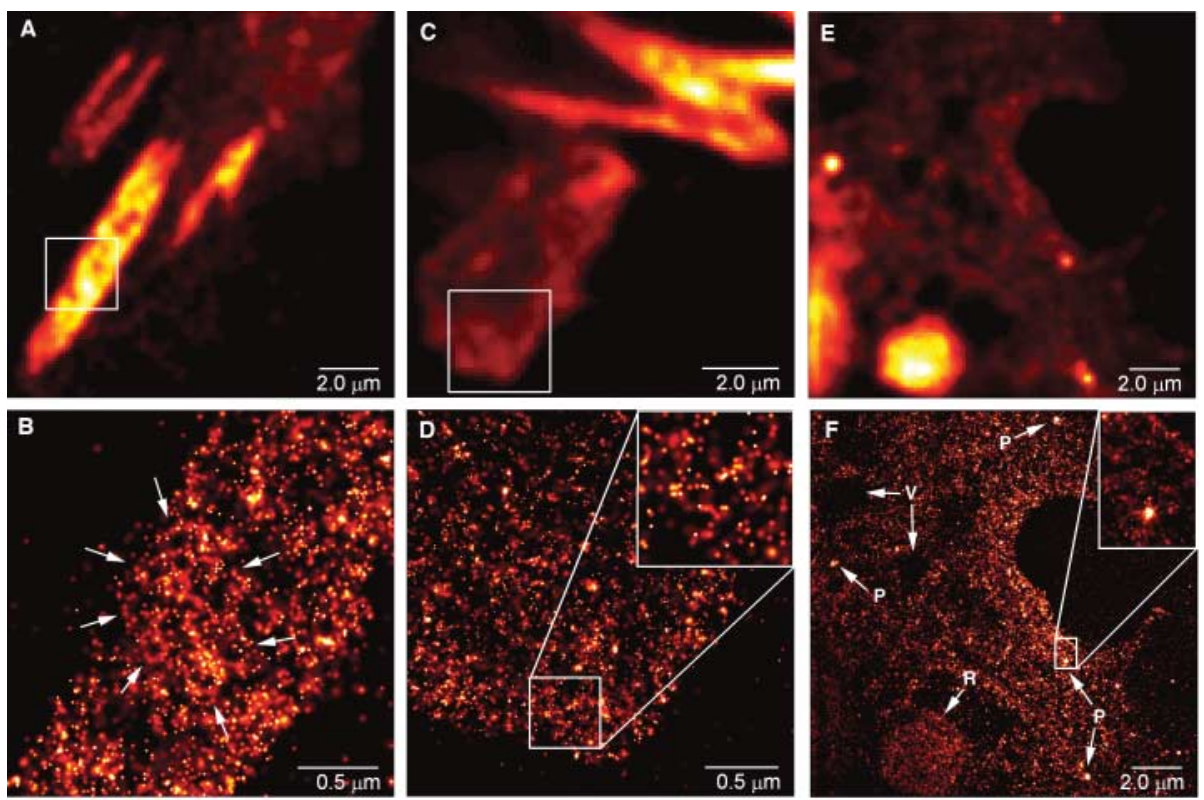

Fig. 4. Examples of PALM imaging near the surfaces of whole, fixed cells. (A) A summed-molecule TIRF image of focal adhesions for a FoLu cell expressing dEos-tagged vinculin. (B) A magnified PALM view of the structure within a single adhesion over the region indicated by the box in (A), including apparent assembly of vinculin in a partial network (arrows). (C) A summed-molecule TIRF image near the periphery of a FoLu cell expressing tdEos-tagged actin. (D) A magnified PALM view of the actin distribution within the portion of the lamellipodium outlined by the box in (C). Inset, a further magnified view near the leading edge over the region indicated by the smaller box. (E and $\mathbf{F}$ ) Summed-molecule TIRF and PALM images, respectively, of a COS-7 cell expressing the retroviral protein Gag tagged with dEos. The PALM image highlights voids (arrows labeled V), a higher density region (arrow R), and probable condensation at several points (arrows labeled P) into VLPs of $\sim 100$ - to 150-nm size (inset). cross-sections, and improved stability expected ( 8 ). On the other hand, the ambient, TIRF-based PALM system demonstrated here has the advantage of simplicity, requiring only a TIRFcapable microscope with appropriate lasers, filters, and EMCCD camera, as well as basic acquisition, localization, and image rendering software. As such, it could be widely adopted in short order for the near-molecular resolution imaging of specified proteins for in vitro preparations and fixed cells.

\section{References and Notes}

1. J. Lippincott-Schwartz, G. H. Patterson, Science 300, 87 (2003).

2. E. Betzig, J. K. Trautman, Science 257, 189 (1992).

3. K. I. Willig, S. O. Rizzoli, V. Westphal, R. Jahn, S. W. Hell, Nature 440, 935 (2006).

4. M. G. L. Gustafsson, J. Microsc. 198, 82 (2000)

5. M. G. L. Gustafsson, Proc. Natl. Acad. Sci. U.S.A. 102, 13081 (2005).

6. M. Hofmann, C. Eggeling, S. Jakobs, S. W. Hell, Proc. Natl. Acad. Sci. U.S.A. 102, 17565 (2005)

7. E. Betzig, R. J. Chichester, Science 262, 1422 (1993).

8. W. E. Moerner, J. Phys. Chem. B 106, 910 (2002).

9. H. F. Hess, E. Betzig, T. D. Harris, L. N. Pfeiffer, K. W. West, Science 264, 1740 (1994)

10. E. Betzig, Opt. Lett. 20, 237 (1995).

11. M. K. Cheezum, W. F. Walker, W. H. Guilford, Biophys. J. 81, 2378 (2001)

12. R. E. Thompson, D. R. Larson, W. W. Webb, Biophys. J. 82, 2775 (2002).

13. A. Yildiz et al., Science 300, 2061 (2003); published online 5 June 2003 (10.1126/science.1084398)

14. X. Qu, D. Wu, L. Mets, N. F. Scherer, Proc. Natl. Acad. Sci. U.S.A. 101, 11298 (2004).

15. L. S. Churchman, Z. Ökten, R. S. Rock, J. F. Dawson, J. A. Spudich, Proc. Natl. Acad. Sci. U.S.A. 102, 1419 (2005).

16. A. M. van Oijen, J. Köhler, J. Schmidt, M. Müller, G. J. Brackenhoff, J. Opt. Soc. Am. A 16, 909 (1999).

17. M. P. Gordon, T. Ha, P. R. Selvin, Proc. Natl. Acad. Sci. U.S.A. 101, 6462 (2004).

18. K. A. Lidke, B. Rieger, T. M. Jovin, R. Heintzmann, Opt Express 13, 7052 (2005)

19. G. H. Patterson, J. Lippincott-Schwartz, Science 297, 1873 (2002).

20. R. Ando, H. Hama, M. Yamamoto-Hino, H. Mizuno, A. Miyawaki, Proc. Natl. Acad. Sci. U.S.A. 99, 12651 (2002).

21. J. Wiedenmann et al., Proc. Natl. Acad. Sci. U.S.A. 101, 15905 (2004).

22. R. Ando, H. Mizuno, A. Miyawaki, Science 306, 1370 (2004).

23. H. Tsutsui, S. Karasawa, H. Shimizu, N. Nukina, A. Miyawaki, EMBO Rep. 6, 233 (2005).

24. K. A. Lukyanov, D. M. Chudakov, S. Lukyanov, V. V. Verkhusha, Nat. Rev. Mol. Cell Biol. 6, 885 (2005).

25. Materials and methods are available as supporting material on Science Online.

26. D. Axelrod, Methods Cell Biol. 30, 245 (1989).

27. G. Gaietta et al., Science 296, 503 (2002).

28. J. C. Politz, Trends Cell Biol. 9, 284 (1999).

29. I. Chen, A. Y. Ting, Curr. Opin. Biotechnol. 16, 35 (2005)

30. E. Betzig, Opt. Express 13, 3021 (2005).

31. This project was supported in part by the Intramural Program of NICHD and the NIH Intramural AIDS Targeted Antiviral Program (IATAP). We thank J. Wiedenmann and U. Nienhaus of the University of Ulm for the gift of a cDNA encoding the EosFP.

\section{Supporting Online Material}

www.sciencemag.org/cgi/content/full/1127344/DC1

Materials and Methods

Figs. S1 to $\mathrm{S} 10$

Table S1

Movie S1

References

13 March 2006; accepted 2 August 2006

Published online 10 August 2006;

10.1126/science. 1127344

Include this information when citing this paper. 